\title{
A Novel Prognostic Nomogram for Predicting Risks of Distant Failure in Patients with Invasive Breast Cancer Following Postoperative Adjuvant Radiotherapy
}

\author{
Yu Jin Lim, $M D^{1}$ \\ Sea-Won Lee, MD ${ }^{1}$ \\ Noorie Choi, MD' \\ Jeanny Kwon, MD, PhD' \\ Keun-Yong Eom, MD, PhD 1,2 \\ Eunyoung Kang, MD, $\mathrm{PhD}^{2}$ \\ Eun-Kyu Kim, MD, PhD² \\ Jee Hyun Kim, MD, PhD2 \\ Yu Jung Kim, MD, $P h D^{2}$ \\ Se Hyun Kim, MD, PhD2 \\ So Yeon Park, MD, PhD² \\ In Ah Kim, MD, PhD 1,2
}

${ }^{1}$ Department of Radiation Oncology,

Seoul National University Bundang Hospital, Seongnam, ${ }^{2}$ Breast Care Center,

Seoul National University Bundang Hospital,

Seoul National College of Medicine,

Seongnam, Korea

\section{Purpose}

This study aimed to identify predictors for distant metastatic behavior and build a related prognostic nomogram in breast cancer.

\section{Materials and Methods}

A total of 1,181 patients with non-metastatic breast cancer between 2003 and 2011 were analyzed. To predict the probability of distant metastasis, a nomogram was constructed based on prognostic factors identified using a Cox proportional hazards model.

\section{Results}

The 7-year overall survival and 5-year post-progression survival of locoregional versus distant recurrence groups were $67.6 \%$ versus $39.1 \%(p=0.027)$ and $54.2 \%$ versus $33.5 \%$ $(p=0.043)$, respectively. Patients who developed distant metastasis showed early and late mortality risk peaks within 3 and after 5 years of follow-up, respectively, but a broad and low risk increment was observed in other patients with locoregional relapse. In multivariate analysis of distant metastasis-free interval, age ( $\geq 45$ years vs. $<45$ years), molecular subtypes (luminal A vs. luminal B, human epidermal growth receptor 2, and triple negative), T category (T1 vs. T2-3 and T4), and N category (NO vs. N1 and N2-3) were independently associated ( $p<0.05$ for all). Regarding the significant factors, a well-validated nomogram was established (concordance index, 0.812). The risk score level of patients with initial brain failure was higher than those of non-brain sites $(p=0.029)$.

\section{Conclusion}

The nomogram could be useful for predicting the individual probability of distant recurrence in breast cancer. In high-risk patients based on the risk scores, more aggressive systemic therapy and closer surveillance for metastatic failure should be considered.
Correspondence: In Ah Kim, MD, PhD

Department of Radiation Oncology,

Seoul National University Bundang Hospital,

82 Gumi-ro 173beon-gil, Bundang-gu,

Seongnam 13620, Korea

Tel: 82-31-787-7651

Fax: 82-31-787-4019

E-mail: inah228@snu.ac.kr

Received October 24, 2017

Accepted December 5, 2017

Published Online December 7, 2017

\section{Key words}

Breast neoplasms, Adjuvant radiotherapy, Nomogram, Neoplasm metastasis, Prognosis

\section{Introduction}

According to recent statistics in the United States, breast cancer is most common in women who are newly diagnosed with malignant tumors [1]. Although systemic treatment based on gene expression profiling has been generalized in clinics, breast cancer is still a leading overall cause of cancer deaths in women [1]. Regarding the wide spectrum of bio- logical characteristics of the malignancy, current guidelines recommend different therapeutic approaches according to initial tumor extent and differential expression status of the hormone receptor and human epidermal growth receptor 2 (HER2) [2,3]. Nevertheless, the potential of distant recurrence remains a major challenge in breast cancer. Advances in early radiological detection methods and novel chemotherapeutic drugs have improved the prognoses after distant tumor progression, but the median survival time of such cases ranges 
from a few months to approximately 3 years [4].

In a recent population-based analysis, approximately $7 \%$ of overall breast cancer patients developed distant metastasis [5]. The metastatic capacity is affected by the natural course of the disease and the intrinsic tendency of spreading tumor cells [6]. A more aggressive nature results in further tumor spread, which leads to differential long-term mortality [7]. Because conventional anti-cancer treatment is limited in eradicating the metastatic tumor cells completely, identifying high-risk patients who can benefit from an appropriate treatment of overt micrometastases is a priority. Considering the relatively longer survival duration of breast cancer patients, sufficiently long-term follow-up data are required for the outcome analysis.

Given the prior investigation of our institution [8], we evaluated the prognostic impact of initial failure types in breast cancer patients who underwent surgery plus postoperative radiotherapy (RT). Distinct time-course change patterns of mortality risk according to locoregional and distant recurrences were evaluated. Prognostic factors for distant metastasis were assessed, and a novel nomogram to predict the risk of distant tumor relapse was developed and validated. The present study was based on contemporary subtypebased systemic and / or endocrine treatments. Therefore, our scoring system may help to differentiate breast cancer patients with higher metastatic potential in a routine clinical evaluation.

\section{Materials and Methods}

\section{Study population}

We analyzed a total of 1,181 breast cancer patients who completed surgery plus postoperative adjuvant RT at Seoul National University Bundang Hospital from January 2003 to December 2011. The eligibility criteria included: (1) initially M0 category, (2) no previous history of malignancy, (3) no refusal of systemic and/or endocrine treatment, and (4) at least 1 year of follow-up. Demographic and clinicopathological information with survival and recurrence data were collected from our medical records database.

\section{Molecular subtypes and treatments}

We evaluated the expression status of estrogen receptor (ER), progesterone receptor (PR), HER2, and Ki-67 to determine molecular subtypes of luminal A (LA), luminal B (LB), HER2, and triple negative (TN), according to the 2013 St. Gallen Consensus criteria [9]. Given the immunohistochem- istry (IHC) results, HER2-positive status was defined as: (1) c-erbB2 overexpression with an IHC score of 3, and (2) the presence of HER2 gene amplification on fluorescence in situ hybridization with a c-erbB2 IHC score of 2 . The expression of $\mathrm{Ki}-67$ index was classified into a low $(<14 \%)$ or high $(\geq 14 \%)$ level. The definition of each subtype was as follows: LA for ER(+), PR(+), HER2(-), and a low level of Ki-67 index; LB for $\operatorname{ER}(+)$ with at least a high Ki-67 labeling index, PR(-), or HER2(+); HER2 for ER(-), PR(-), and HER2(+); TN for ER(-), PR(-), and HER2(-), respectively. The seventh edition of the American Joint Committee on Cancer (AJCC) staging system was used to assess primary tumor stage and lymph node status. For staging of patients with neoadjuvant chemotherapy prior to surgery, clinical $\mathrm{T}$ and $\mathrm{N}$ information was applied. All patients underwent curative surgery followed by postoperative adjuvant RT. Elective RT for supraclavicular or internal mammary nodal areas was determined based on the extent of nodal disease at diagnosis. Regarding the contemporary guideline based on molecular status and patient and/or tumor-related factors, clinicians determined the use of endocrine and/or HER2-targeted agents for each patient.

\section{Follow-up data}

Post-treatment initial failure events were categorized as locoregional and distant metastatic recurrences. Locoregional recurrence was defined as recurred tumors at the ipsilateral breast/chest wall or regional lymphatics. Distant metastatic events included lymphatic spread other than ipsilateral regional lymph nodes, and tumor relapse beyond the locoregional sites, such as lung and/or pleura, visceral organs, bone, and the central nervous system.

\section{Statistical analysis}

Overall survival (OS) was defined as the time period between the start date of treatment and overall deaths. Postprogression survival (PPS) was the time interval between the diagnosis of initial tumor recurrence and overall deaths. Distant metastasis-free interval (DMFI) was estimated based on distant metastatic events as the first post-treatment failure. The Kaplan-Meier method and log-rank test were used to assess differential outcomes. The Cox proportional hazards model was used for multivariate analysis of DMFI. The proportional hazards assumptions were confirmed using logminus-log survival plots. Potentially significant factors to be included in multivariate analysis were selected applying the Akaike's Information Criteria [10]. According to the final set of the Cox regression model, a prognostic nomogram to predict risks of distant metastatic failure was developed. The accuracy of the prognostic model was evaluated using the 
concordance index. The calibration plot represented the predicted and actual 10-year probability of distant metastasis on the $x$ - and $y$-axis, respectively, which was used to assess the calibration rate. Two-sided p-values $<0.05$ were considered statistically significant. SPSS ver. 22 (IBM Corp., Armonk, NY) and R ver. 3.2.3 (https:// www.r-project.org) were used for all statistical analyses.

\section{Ethical statement}

This study was approved by the Institutional Review Board (IRB) of our institution (IRB No: B-1505/298-116). The necessity of informed consent for this retrospective analysis was waived.

\section{Results}

\section{Patient characteristics}

S1 Table presents the patient characteristics $(n=1,181)$. Younger age ( $<45$ years) was observed in 382 patients $(32 \%)$. The LA, LB, HER2, and TN subtypes were verified in $38 \%$, $33 \%, 10 \%$, and $19 \%$ of the patients, respectively. T1, T2, T3, and T4 category was diagnosed in $615(52 \%), 428(36 \%), 102$ $(9 \%)$, and $36(3 \%)$ patients, respectively, and node-positive disease was observed in $529(45 \%)$ patients. Histologic grade III and presence of lymphovascular invasion were reported in $379(32 \%)$ and $425(36 \%)$ patients, respectively. Breast-conserving surgery and mastectomy were performed to 936 $(79 \%)$ and $245(21 \%)$ patients, respectively. In LA $(\mathrm{n}=446)$ and LB $(n=384)$ groups, $439(98 \%)$ and $362(94 \%)$ patients were treated with endocrine treatment. Chemotherapy was administered in 928 patients (79\%). Of them, adjuvant, neoadjuvant, and both adjuvant and neoadjuvant chemotherapy were administered in 664, 152, and 112 patients, respectively. Regarding the systemic treatment methods according to molecular subtypes, $53 \%$ and $12 \%, 71 \%$ and $24 \%, 68 \%$ and $41 \%$, and $80 \%$ and $29 \%$ of LA, LB, HER2, and TN patients were treated with adjuvant and neoadjuvant chemotherapy, respectively. When the patients were categorized according to the year of diagnosis, such as " $<2006$ vs. $\geq 2006$," "< 2007 vs. $\geq 2007$," and " $<2008$ vs. $\geq 2008$," the proportion of adriamycin use was higher in patients who were diagnosed later in time ( $77 \%$ vs. $85 \%, 78 \%$ vs. $85 \%$, and $78 \%$ vs. $86 \%$ with $\mathrm{p}=0.014, \mathrm{p}=0.017$, and $\mathrm{p}=0.001$, respectively). However, the year of diagnosis $(\mathrm{p}=0.155, \mathrm{p}=0.215$, and $p=0.524$ on the basis of the year of 2006, 2007, and 2008, respectively) did not affect DMFI.

\section{Outcome analysis}

The median follow-up duration was 76 months. When the patients were categorized according to the initial failure patterns, locoregional and distant metastatic recurrence was observed in 29 and 81 patients, respectively. The organ sites of distant tumor spread included: bone $(\mathrm{n}=28)$, lung and / or pleura $(\mathrm{n}=17)$, distant lymph nodes $(\mathrm{n}=12)$, liver $(\mathrm{n}=17)$, and

A
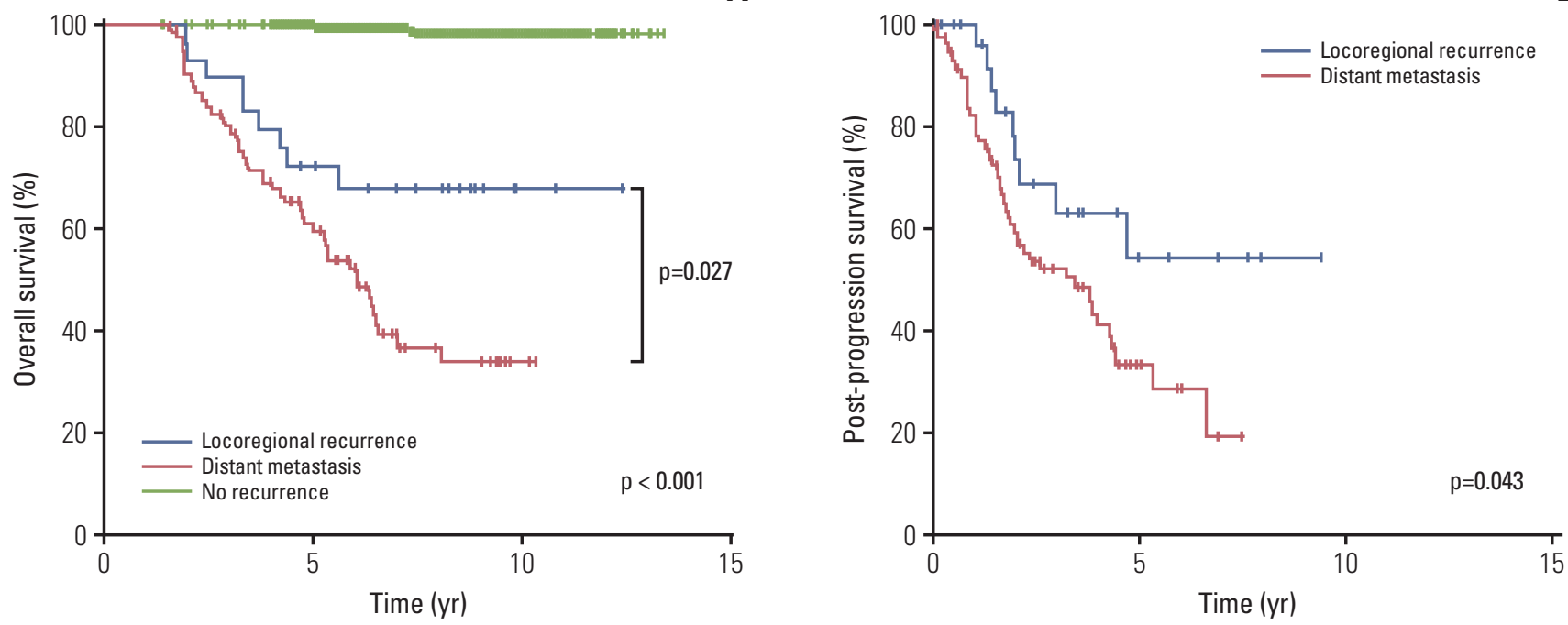

Fig. 1. Survival time according to the initial patterns of failure after postoperative radiotherapy: overall survival (A) and post-progression survival (B). 


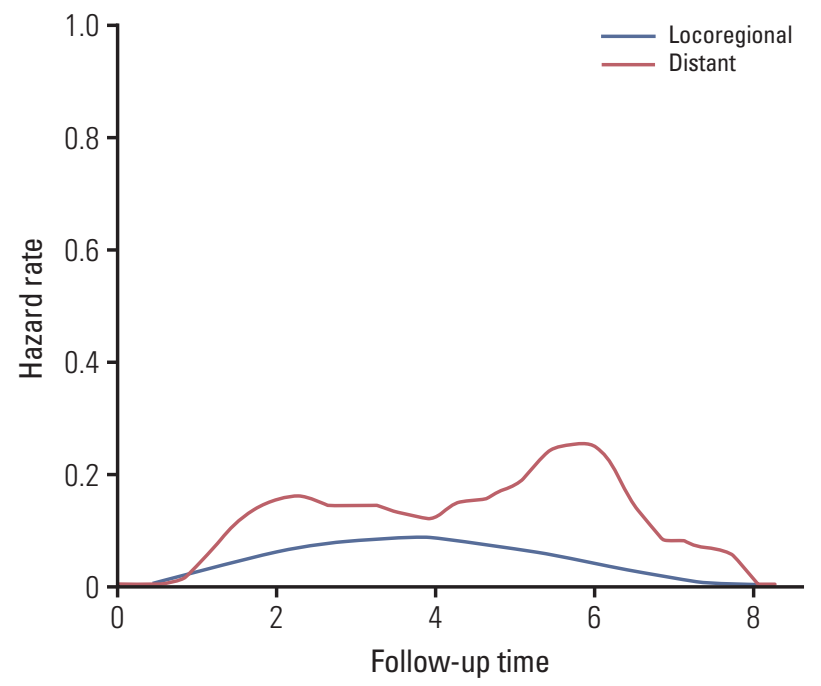

Fig. 2. Baseline hazard function plot of overall survival according to the initial pattern of failure after postoperative radiotherapy.

brain and/or leptomeningeal seeding $(\mathrm{n}=7)$.

The 7-year OS rates for patients with no recurrence, locoregional recurrence, and distant metastasis were $99.1 \%, 67.6 \%$, and $39.1 \%$, respectively $(\mathrm{p}<0.001)$ (Fig. 1A). The survival difference between the locoregional and distant recurrence groups was statistically significant $(p=0.027)$. Estimating the survival time after recurred events, the 5-year rates of PPS were $54.2 \%$ and $33.5 \%$ for patients with locoregional recurrence and distant metastasis, respectively ( $\mathrm{p}=0.043$ ) (Fig. 1B). Baseline hazard rate function plots of mortality according to the failure patterns are represented in Fig. 2. In patients with distant metastasis, the mortality risk initially increased within 3 years, and the risk surge appeared again after 5 years of follow-up. However, in patients with locoregional recurrence, a relatively low and continuous risk increase was observed.

\section{Prognostic factors for distant metastatic failure}

Table 1 shows the significant prognostic factors associated with DMFI. The 10-year percentages of DMFI were significantly different according to age ( $<45$ years and $\geq 45$ years; $88.1 \%$ and $94.4 \%$, respectively; $\mathrm{p}=0.002)$, molecular subtypes (LA, LB, HER2, and TN; 96.8\%, 91.1\%, 86.5\%, and 88.9\%, respectively; $\mathrm{p}<0.001)$, $\mathrm{T}$ category (T1, $\mathrm{T} 2-3$, and $\mathrm{T} 4 ; 98.1 \%$, $86.8 \%$, and $76.3 \%$, respectively; $\mathrm{p}<0.001$ ), $\mathrm{N}$ category (N0, $\mathrm{N} 1$, and $\mathrm{N} 2-3 ; 98.0 \%, 92.0 \%$, and $77.7 \%$, respectively; $\mathrm{p}<0.001$ ), histological grade (I-II and III; $92.7 \%$ and $90.4 \%$, respectively; $\mathrm{p}<0.001$ ), lymphovascular invasion (no and yes; $96.0 \%$ and $86.9 \%$, respectively; $\mathrm{p}<0.001$ ), types of pri- mary surgery (breast-conserving and mastectomy; $96.3 \%$ and $77.5 \%$, respectively; $\mathrm{p}<0.001$ ), lymph node dissection (no and yes; $97.8 \%$ and $86.1 \%$, respectively; $\mathrm{p}<0.001$ ), and use of chemotherapy (no and yes; $98.8 \%$ and $90.5 \%$, respectively; $\mathrm{p}<0.001$ ). Given the result of univariate analysis, the multivariate Cox proportional hazards model indicated independently significant factors to be included in a prognostic nomogram as follows: age ( $<45$ years vs. $\geq 45$ years; hazard ratio $[H R], 0.60 ; p=0.049$ ), molecular subtypes (LA vs. LB, HER2, and TN; HR 2.25, 4.50, and 5.13, respectively; $\mathrm{p}=0.034$, $\mathrm{p}=0.001$, and $\mathrm{p}<0.001$, respectively), T category (T1 vs. T2-3 and T4; HR, 2.27 and 3.68; $p=0.023$ and $p=0.010$, respectively), and N category (N0 vs. N1 and N2-3; HR, 3.44 and 6.98; $\mathrm{p}=0.043$ and $\mathrm{p}=0.002$, respectively).

S2 Table and S3 Fig. show the subgroup analysis of OS in patients with distant metastasis $(\mathrm{n}=81)$. Among the patient, tumor, and treatment-related variables, molecular subtypes (luminal, HER2, and TN; $84.6 \%, 60.0 \%$, and $23.3 \%$, respectively; $\mathrm{p}<0.001$ ) and histological grade (I-II and III; 75.8\% and $43.2 \%$; $\mathrm{p}=0.005$ ) were associated with different 5 -year OS rates. However, other factors were not significant.

In terms of the timing of chemotherapy, the 10-year DMFI rates of adjuvant, neoadjuvant, and both adjuvant and neoadjuvant chemotherapy were $94.8 \%, 79.1 \%$, and $79.2 \%$, respectively, with statistical significances in paired comparisons of adjuvant vs. neoadjuvant $(\mathrm{p}<0.001)$ and adjuvant vs. both treatment $(\mathrm{p}<0.001)$ groups. However, the distribution of clinicopathologic characteristics was imbalanced according to the timing of chemotherapy. Patients with adjuvant chemotherapy had more favorable features in terms of $\mathrm{T} / \mathrm{N}$ category and lymphovascular invasion (S4 Table).

\section{A nomogram for predicting distant metastasis}

Regarding the prognostic factors (age, molecular subtypes, $\mathrm{T}$ category, and $\mathrm{N}$ category), a prognostic nomogram for predicting distant metastatic failure after surgery plus postoperative RT was developed (Fig. 3). The concordance index for this model was 0.812 . The plot representing the predicted and actual Kaplan-Meier DMFI probability at 10 years shows the well-calibrated results (Fig. 4).

\section{Risk scores according to metastatic sites}

According to our nomogram, risk scores were calculated for each patient with distant metastasis. When the scores were compared between the groups of brain $(n=7)$ vs. nonbrain $(n=74)$ metastases, the level of risk scores of patients with metastatic tumor spread to brain was significantly higher than the others (mean values of 21.5 vs. 17.8, respectively; $\mathrm{p}=0.029$ ) (Fig. 5). 
Table 1. Prognostic factors for distant metastasis-free interval

\begin{tabular}{|c|c|c|c|c|}
\hline \multirow{2}{*}{ Variable } & \multicolumn{2}{|c|}{ Univariate } & \multicolumn{2}{|c|}{ Multivariate } \\
\hline & $10-Y e a r$ rate $(\%)$ & p-value & $\mathrm{HR}(95 \% \mathrm{CI})$ & p-value \\
\hline \multicolumn{5}{|l|}{ Age (yr) } \\
\hline$<45$ & 88.1 & 0.002 & 1 & \\
\hline$\geq 45$ & 94.4 & & $0.60(0.39-0.99)$ & 0.049 \\
\hline \multicolumn{5}{|l|}{ Menopausal status } \\
\hline Premenopausal & 91.2 & 0.141 & & \\
\hline Perimenopausal or postmenopausal & 94.1 & & & \\
\hline \multicolumn{5}{|l|}{ Molecular subtype } \\
\hline Luminal A & 96.8 & $<0.001$ & 1 & \\
\hline Luminal B & 91.1 & & $2.25(1.06-4.77)$ & 0.034 \\
\hline HER2 & 86.5 & & $4.50(1.78-11.40)$ & 0.001 \\
\hline Triple negative & 88.9 & & $5.13(2.20-11.95)$ & $<0.001$ \\
\hline \multicolumn{5}{|l|}{ T category } \\
\hline $\mathrm{T} 1$ & 98.1 & $<0.001$ & 1 & \\
\hline $\mathrm{T} 2-3$ & 86.8 & & $2.27(1.12-4.58)$ & 0.023 \\
\hline $\mathrm{T} 4$ & 76.3 & & $3.68(1.37-9.87)$ & 0.010 \\
\hline \multicolumn{5}{|l|}{$\mathrm{N}$ category } \\
\hline N0 & 98.0 & $<0.001$ & 1 & \\
\hline N1 & 92.0 & & $3.44(1.04-11.37)$ & 0.043 \\
\hline N2-3 & 77.7 & & $6.98(2.06-23.62)$ & 0.002 \\
\hline \multicolumn{5}{|l|}{ Histologic grade } \\
\hline I-II & 92.7 & $<0.001$ & 1 & \\
\hline III & 90.4 & & $0.76(0.43-1.32)$ & 0.329 \\
\hline \multicolumn{5}{|l|}{ Lymphovascular invasion } \\
\hline No & 96.0 & $<0.001$ & 1 & \\
\hline Yes & 86.9 & & $1.23(0.71-2.14)$ & 0.466 \\
\hline \multicolumn{5}{|l|}{ Primary surgery } \\
\hline Breast-conserving & 96.3 & $<0.001$ & 1 & \\
\hline Mastectomy & 77.5 & & $1.21(0.41-3.59)$ & 0.733 \\
\hline \multicolumn{5}{|l|}{ Lymph node surgery } \\
\hline No ALND & 97.8 & $<0.001$ & 1 & \\
\hline ALND & 86.1 & & $1.05(0.36-3.09)$ & 0.933 \\
\hline \multicolumn{5}{|l|}{ Chemotherapy } \\
\hline No & 98.8 & $<0.001$ & 1 & \\
\hline Yes & 90.5 & & $0.83(0.23-2.99)$ & 0.778 \\
\hline
\end{tabular}

$\mathrm{HR}$, hazard ratio; CI, confidence interval; HER2, human epidermal growth factor receptor 2; ALND, axillary lymph node dissection.

\section{Discussion}

This study evaluated differential OS and PPS according to locoregional and distant metastatic spread as the initial patterns of failure in breast cancer. The baseline hazard rate function plot of the distant metastasis group showed early and late mortality risk peaks, whereas there was a low and broad risk increment in cases of locoregional relapse. Regarding the prognostic implications of distant tumor progression, we constructed a nomogram to predict distant metastatic failure risk in contemporary clinical practice. Age, molecular subtypes, and $\mathrm{T}$ and $\mathrm{N}$ category at diagnosis were the independently associated determinants, and the nomogram was a well-validated model. Calculating total score points based on the nomogram, we observed higher level of risk scores with brain metastasis, in comparison with those of non-brain metastases.

We verified the poor prognostic impact of distant metastatic behavior on the long-term OS and PPS. As well as the 


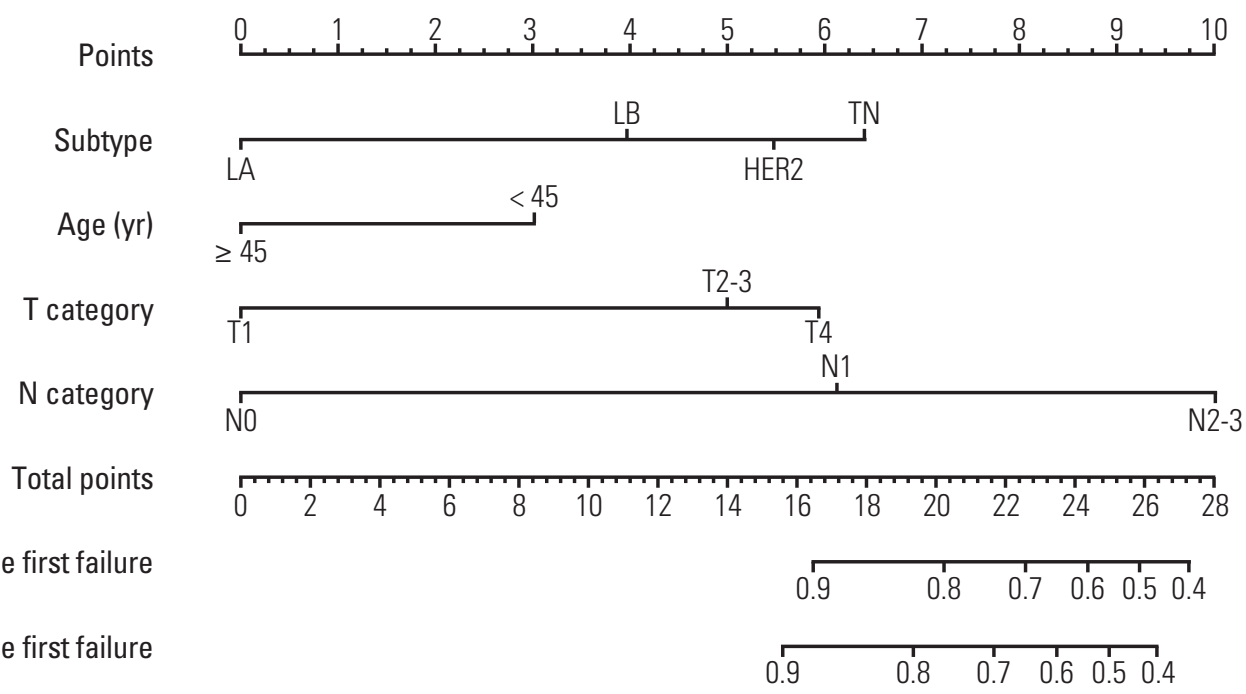

Fig. 3. Nomogram predicting distant metastatic failure. LA, luminal A; LB, luminal B; HER2, human epidermal growth factor receptor 2; TN, triple negative; DMFI, distant metastasis-free interval.

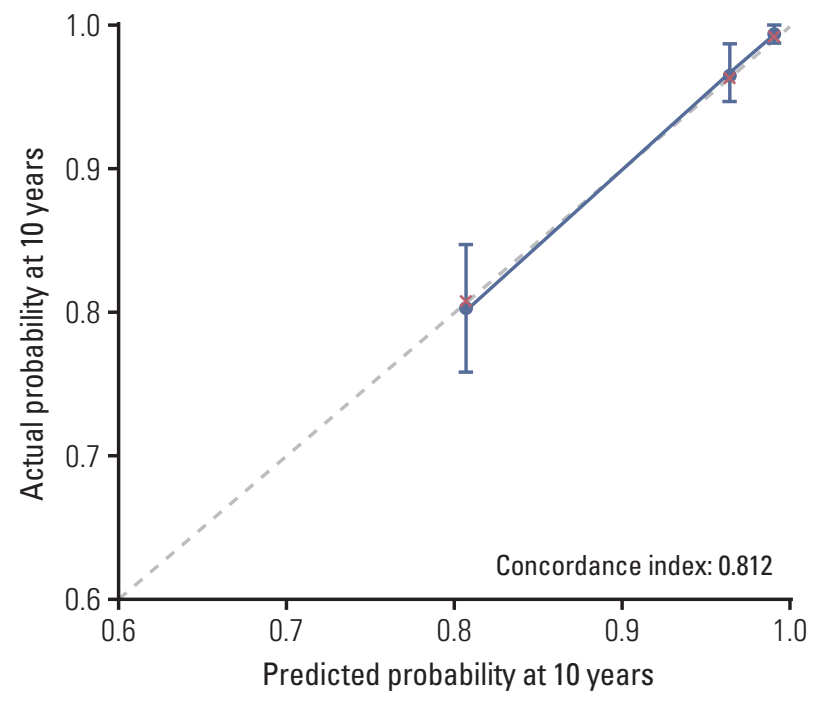

Fig. 4. Calibration plot for comparison of predicted and observed 10-year rates of distant metastasis-free interval.

intrinsic aggressiveness of metastatic tumor cells, limited systemic treatment options for clinical cure contribute to higher mortality risk after distant tumor progression [11]. Additionally, the distinct early and late risk peaks of overall deaths in patients with distant failure were different from the indolent pattern of risk increase in the group with locoregional recurrences. The short-term peak that emerged within 3 years indicated the lethality and highly aggressive character of the tumor cells. In addition, the limited therapeutic effects or

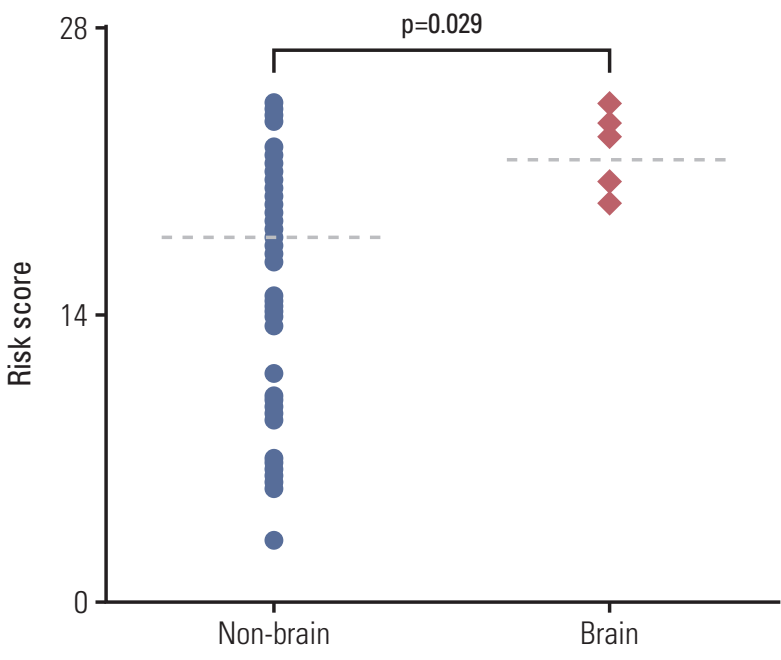

Fig. 5. Comparison of risk scores according to different metastatic sites (dotted lines: median values).

resistance to conventional systemic treatment under the palliative setting might increase death events even after 5 years of follow-up. Therefore, an early therapeutic intervention to eradicate overt micrometastases is important to improve long-term prognosis of breast cancer.

Nomograms are known as representative tools for calculating the probability of specific outcomes in individual patients [12]. The two-dimensional diagram consists of a set of statistically significant factors associated with the risk of 
an event of interest. In the clinical practice, the graphical format is used to predict the occurrence of events. In our previous institutional analysis of patients who completed curative locoregional treatments, tumor relapse at the primary breast/chest wall and regional lymphatic pathways were relatively low, whereas distant metastatic risk was significantly different according to major molecular subtypes [8]. Although other prior studies have analyzed long-term outcomes and failure patterns of breast cancer $[13,14]$, risk factors strongly affecting the development of distant metastasis are not completely understood. Therefore, we established a statistical model to identify patients who are susceptible to distant metastasis after completion of the multimodality treatment in breast cancer. Because this study included patients treated under the uniform and contemporary clinical practices, treatment strategies and post-treatment surveillance methods were consistent. Additionally, the present long-term data could sufficiently detect the late occurrence of recurred events. When estimating distant metastatic failure risks after subtype-based curative treatment, our nomogram provides guidance to an individual-based systemic approach for better prognosis in breast cancer.

It was expected that young age would be associated with a higher risk of failure in breast cancer, but its impact on long-term survival is still controversial [15-17]. In a largescale institutional study, distant metastatic risk significantly decreased in patients older than 40 years, which was similar with our results [18]. The HR values for overall metastatic sites ranged from approximately $0.6-0.8$ in ages $>40$ years, in comparison with the risk of those $<40$ years of age. The protective effect of old age on distant metastatic tumor progression might be because of age-induced changes of the host immune system or the tumor microenvironment involved in the migration of tumor cells $[19,20]$. However, old age can be a disadvantageous factor in that more intensive systemic treatment is often not medically feasible because of poor performance status or combined medical comorbidities [21]. Because the present study population received systemic and / or endocrine therapy based on physicians' discretion and guidelines, this study suggests the adverse effects of young age associated with distant tumor dissemination.

On the basis of our prior analysis [8], the present prognostic nomogram showing the differential contribution of the molecular subtypes is an informative predictor of metastatic behavior. One interesting point was the different risk scores within the luminal tumors, between the LA and LB subtypes. Similarly, a study by Arriagada et al. [22] suggested that the PR status was an independent risk factor (relative hazard value of 0.8 for a higher PR level; $\mathrm{p}=0.003$ in multivariate analysis) affecting the likelihood of metastatic dissemination. It has been suggested that more complicated categories beyond the major molecular subtypes exist in breast cancer
[23]. Especially within the luminal tumors, heterogeneous characteristics in relation to biological aggressiveness and responsiveness to hormonal therapy have been observed [24]. We suggest that comprehensive information regarding the ER, PR, HER2, and Ki-67 status is needed to estimate the risk of distant tumor relapse. Although subclassification of LB-HER2(-) and LB-HER2(+) within the luminal tumors was not considered significantly prognostic in our nomogram, multi-institutional large-scale analysis is needed to assess the potential of differential risk level.

In recent years, cumulative development of brain metastasis has been estimated at approximately $10-30 \%$ of overall breast cancer [25]. As well as the aging of patient population, enhanced control of systemic tumor cells and radiologic imaging techniques also contributed to early detection of brain metastasis [26]. Nevertheless, brain metastasis is one of the fatal causes of death in breast cancer, showing the worst survival outcome in comparison with other metastatic sites [27]. In the similar context, our nomogram-based risk calculation demonstrated that patients with initial brain metastasis represented higher risk scores than those of the other metastatic sites. This result might be related to the heterogeneity in the propensity to metastatic dissemination within the patients with distant failure [28]. Nevertheless, due to the small number of initial brain metastasis events $(n=7)$, our results should be interpreted with caution. Further studies need to elucidate the differential risk level within the patients with distant failure, suggesting the potential of brain metastasis.

This study has some limitations. Because of the retrospective design, the potential of selection bias cannot be excluded. Although our nomogram showed well-validated results, further validation using other institutional data are necessary. We excluded patients who refused their planned or recommended treatment, but the temporal change of chemotherapy regimens could not be adjusted. Beyond the clinicopathological prognostic factors, the impact of genomics data on differential prognosis of breast cancer has been reported in recent years [29]. Therefore, future prediction models need to integrate biological characteristics of tumor cells and the tumor microenvironment.

We evaluated differential prognostic implications according to initial patterns of failure in breast cancer patients undergoing surgery plus postoperative adjuvant RT. Distant metastatic tumor spread led to higher overall mortality, in comparison with locoregional relapse. Distinct early and late mortality risk peaks on baseline hazard rate function plots were characteristic of the patients who developed distant failures. On the basis of significant prognostic factors associated with DMFI, this study established a nomogram to predict the likelihood of individual metastatic behavior. The present analysis could be informative to identify a subset of 
patients with potential overt micrometastases, suggesting the need of personalized chemotherapeutic strategies. Close surveillance for metastatic failure should be considered for patients with relatively higher risk scores.

\section{Electronic Supplementary Material}

Supplementary materials are available at Cancer Research and Treatment website (http:// www.e-crt.org).

\section{Conflicts of Interest}

Conflict of interest relevant to this article was not reported.

\section{Acknowledgments}

This work was supported by the grants from Korean Ministry of Science and Information \& Communication Technology to Kim IA (No. 2017R1A2B4002710 \& 2017M2A2A7A01018438). Lim YJ, Lee SW, and Kwon J are currently work at Kyung Hee University Medical Center, Seoul St. Mary's Hospital, and Chungnam National University Hospital, respectively.

\section{References}

1. Siegel RL, Miller KD, Jemal A. Cancer statistics, 2016. CA Cancer J Clin. 2016;66:7-30.

2. National Comprehensive Cancer Network. Breast cancer version 2.2017 [Internet]. Fort Washington, PA: National Comprehensive Cancer Network; c2017 [cited 2017 Oct 23]. Available from: http://www.nccn.org/professionals/ physician_gls/pdf/breast.pdf.

3. National Institute for Health and Care Excellence. Adjuvant therapy for early and locally advanced breast cancer [Internet]. London: National Institute for Health and Care Excellence; c2017 [cited 2017 Oct 23]. Available from: http:// pathways. nice.org.uk/ pathways / early-and-locally-advanced-breastcancer.

4. Wu SG, Li H, Tang LY, Sun JY, Zhang WW, Li FY, et al. The effect of distant metastases sites on survival in de novo stageIV breast cancer: a SEER database analysis. Tumour Biol. 2017;39:1010428317705082.

5. Wu Q, Li J, Zhu S, Wu J, Chen C, Liu Q, et al. Breast cancer subtypes predict the preferential site of distant metastases: a SEER based study. Oncotarget. 2017;8:27990-6.

6. Weigelt B, Peterse JL, van 't Veer LJ. Breast cancer metastasis: markers and models. Nat Rev Cancer. 2005;5:591-602.

7. Le MG, Arriagada R, Spielmann M, Guinebretiere JM, Rochard F. Prognostic factors for death after an isolated local recurrence in patients with early-stage breast carcinoma. Cancer. 2002;94:2813-20.

8. Lim YJ, Lee SW, Choi N, Kwon J, Eom KY, Kang E, et al. Failure patterns according to molecular subtype in patients with invasive breast cancer following postoperative adjuvant radiotherapy: long-term outcomes in contemporary clinical practice. Breast Cancer Res Treat. 2017;163:555-63.

9. Goldhirsch A, Winer EP, Coates AS, Gelber RD, Piccart-Gebhart $\mathrm{M}$, Thurlimann $\mathrm{B}$, et al. Personalizing the treatment of women with early breast cancer: highlights of the St Gallen International Expert Consensus on the Primary Therapy of Early Breast Cancer 2013. Ann Oncol. 2013;24:2206-23.
10. Akaike H. A new look at the statistical model identification. IEEE Trans Autom Control. 1974;19:716-23.

11. Cheng YC, Ueno NT. Improvement of survival and prospect of cure in patients with metastatic breast cancer. Breast Cancer. 2012;19:191-9.

12. Balachandran VP, Gonen M, Smith JJ, DeMatteo RP. Nomograms in oncology: more than meets the eye. Lancet Oncol. 2015;16:e173-80.

13. Haffty BG, Yang Q, Reiss M, Kearney T, Higgins SA, Weidhaas J, et al. Locoregional relapse and distant metastasis in conservatively managed triple negative early-stage breast cancer. J Clin Oncol. 2006;24:5652-7.

14. Laurberg T, Lyngholm CD, Christiansen P, Alsner J, Overgaard J. Long-term age-dependent failure pattern after breastconserving therapy or mastectomy among Danish lymphnode-negative breast cancer patients. Radiother Oncol. 2016; 120:98-106

15. Anders CK, Hsu DS, Broadwater G, Acharya CR, Foekens JA, Zhang $Y$, et al. Young age at diagnosis correlates with worse prognosis and defines a subset of breast cancers with shared patterns of gene expression. J Clin Oncol. 2008;26:3324-30.

16. Partridge AH, Hughes ME, Warner ET, Ottesen RA, Wong YN, Edge SB, et al. Subtype-dependent relationship between young age at diagnosis and breast cancer survival. J Clin Oncol. 2016;34:3308-14.

17. van de Water W, Markopoulos C, van de Velde CJ, Seynaeve C, Hasenburg A, Rea D, et al. Association between age at diagnosis and disease-specific mortality among postmenopausal women with hormone receptor-positive breast cancer. JAMA. 2012;307:590-7.

18. Purushotham A, Shamil E, Cariati M, Agbaje O, Muhidin A, Gillett $C$, et al. Age at diagnosis and distant metastasis in breast cancer: a surprising inverse relationship. Eur J Cancer. 2014;50:1697-705.

19. Myers CE, Mirza NN, Lustgarten J. Immunity, cancer and aging: lessons from mouse models. Aging Dis. 2011;2:512-23. 
20. Labat-Robert J, Robert L. The effect of cell-matrix interactions and aging on the malignant process. Adv Cancer Res. 2007;98: 221-59.

21. Hancke K, Denkinger MD, Konig J, Kurzeder C, Wockel A, Herr D, et al. Standard treatment of female patients with breast cancer decreases substantially for women aged 70 years and older: a German clinical cohort study. Ann Oncol. 2010;21: 748-53.

22. Arriagada R, Rutqvist LE, Johansson H, Kramar A, Rotstein S. Predicting distant dissemination in patients with early breast cancer. Acta Oncol. 2008;47:1113-21.

23. Ali HR, Rueda OM, Chin SF, Curtis C, Dunning MJ, Aparicio $\mathrm{SA}$, et al. Genome-driven integrated classification of breast cancer validated in over 7,500 samples. Genome Biol. 2014;15: 431.

24. Ciriello G, Sinha R, Hoadley KA, Jacobsen AS, Reva B, Perou $\mathrm{CM}$, et al. The molecular diversity of Luminal A breast tumors. Breast Cancer Res Treat. 2013;141:409-20.
25. Lin NU, Bellon JR, Winer EP. CNS metastases in breast cancer. J Clin Oncol. 2004;22:3608-17.

26. Niwinska A, Murawska M, Pogoda K. Breast cancer brain metastases: differences in survival depending on biological subtype, RPA RTOG prognostic class and systemic treatment after whole-brain radiotherapy (WBRT). Ann Oncol. 2010;21: 942-8.

27. Kennecke H, Yerushalmi R, Woods R, Cheang MC, Voduc D, Speers $\mathrm{CH}$, et al. Metastatic behavior of breast cancer subtypes. J Clin Oncol. 2010;28:3271-7.

28. Almendro V, Kim HJ, Cheng YK, Gonen M, Itzkovitz S, Argani $\mathrm{P}$, et al. Genetic and phenotypic diversity in breast tumor metastases. Cancer Res. 2014;74:1338-48.

29. Fasching PA, Pharoah PD, Cox A, Nevanlinna H, Bojesen SE, Karn T, et al. The role of genetic breast cancer susceptibility variants as prognostic factors. Hum Mol Genet. 2012;21: 3926-39. 